\title{
HUGE EQUATION ACCURACY FOR SCREENING CHRONIC KIDNEY DISEASE: A PROSPECTIVE STUDY
}

\author{
C.G. Musso ${ }^{1}$, S. Maytin ${ }^{2}$, P. Conti ${ }^{2}$, S. Terrasa $a^{3,4}$, A. Primerano ${ }^{2}$, A. Reynaga ${ }^{3}$, M. Vilas ${ }^{1}$, J. Jauregui ${ }^{1}$
}

\begin{abstract}
Objectives: Chronically reduced glomerular filtration rate (GFR) in old people does not always mean that they suffer from chronic kidney disease (CKD) since their GFR can just be induced by ageing. The HUGE equation has been recently described and validated in Spain for screening CKD without taking into account the patient's GFR value This study evaluated HUGE performance in Argentine patiens. Material \& Method: This was a prospective study to assess the operational characteristics of the HUGE equation for diagnosing CKD (diagnostic accuracy) in an ambulatory adult (age $\geq 18$ years old) population (n: 205) assisted at the ambulatory clinical consult of Hospital Italiano de San Justo, Buenos Aires, Argentina in 2016. Results: It was documented that HUGE equation had a sensitivity of $56.7 \%$ (IC: $37.4 \%-74.5 \%$ ), and a specificity of $95.9 \%$ (IC: $88.6 \%-99.1 \%$ ) for screening CKD in elderly individuals who have an estimated GFR $<45 \mathrm{ml} / \mathrm{min} / 1.73 \mathrm{~m}^{2}$. Additionally, it was found that the screening strategy of combining HUGE with estimated GFR and urinalyses, showed a very good performance for detecting CKD, not only in elderly people but also in young people. Both triple combined CKD screening strategies showed a sensitivity around $86 \%$, and 98\% in young and elderly people, respectively. Conclusion: This study documented that the HUGE equation had and acceptable performance for screening moderate-severe CKD (stages III b-V) in elderly people, and an excellent performance for screening CKD in general population in combination with estimated GFR and urinalyses.
\end{abstract}

Key words: HUGE equation, chronic kidney disease, screening test..

\section{Introduction}

Chronic kidney disease (CKD) is defined by the presence of an alteration in at least one of the following renal variables: decreased glomerular filtration rate, electrolyte disorders of tubular origin, abnormal urinalysis (presence of proteinuria and/or renal hematuria), and abnormal renal ultrasound (decreased renal size, cortical thinning, disappearance of cortico-medullary boundary, polycystosis) for a period longer than three months (1). However, it is often wrongly diagnosed CKD in healthy very old people just because they have a reduced glomerular filtration rate (GFR) secondary to ageing; and this mistake comes from basing CKD diagnosis only on a measured or estimated GFR value $(2,3)$. Consequently, this mistaken diagnosis criteria can lead not only to overdiagnose CKD in healthy elderly, as it was previously mentioned, but also to underdiagnose

1. Ageing Biology Unit; 2. Internal Medicine Division; 3. Family and Community Medicine and Research Department ${ }^{3}-$ Hospital Italiano de Buenos Aires, Argentina; 4. Public Health Department -Instituto Universitario Hospital Italiano. Argentina

Corresponding Author: Carlos G. Musso, Ageing Biology Unit, Hospital Italiano de Buenos Aires, Argentina. carlos.musso@hospitalitaliano.org.ar

Received March 3, 2017

Accepted for publication May 29, 2017
CKD in those patients who suffer from early stages (stages I or II) of chronic nephropathy (3). Because of that, Alvarez-Gregori et al. has recently created (n: 376 volunteers) and validated (n: 111 volunteers) in Spain an equation (HUGE) for detecting CKD regardless patient's GFR value, particularly in the elderly. HUGE equation is based on three variables: hematocrit, uremia and gender, and a positive value (HUGE $\geq 0$ ) means significant risk of suffering from CKD, while a negative value (HUGE $<0$ ) means significant chance of having healthy kidneys (4). HUGE original study showed its best performance for screening CKD in elderly population, achieving a sensitivity of $92.9 \%$ and a specificity of $93.15 \%$ (4). Additionally, in a recent retrospective study, we documented in a group of Argentine patients (n: 371) that HUGE equation had a sensitivity of $83.3 \%$ (IC: 78.2 - $87.7 \%$ ), and a specificity of $82.3 \%$ (IC: $74.0-88.9 \%$ ) for screening CKD in elderly patients (5). Thus, in order to explore more deeply the HUGE equation performance, we decided to reevaluate its accuracy in a prospective Argentine population. 


\section{Material and Methods}

This was a prospective study to assess the operational characteristics of the HUGE equation for diagnosing CKD (diagnostic accuracy) in an ambulatory adult (age $\geq 18$ years old) population (n: 205) assisted at the ambulatory clinical consult of Hospital Italiano de San Justo, province of Buenos Aires (Argentina) in 2016. Exclusion criteria were pregnancy, treatment based on nephrotoxic drugs (non-steroidal anti-inflammatory drugs, chemotherapies, calcineurin inhibitors, etc.), dialysis, refusal to participate, or survival estimated less than three months according to clinical status at the time of evaluation (6).

From each patient the following variables were evaluated and recorded: age, gender, body weight, medical history, hematocrit, hemoglobin, serum urea, sodium, potassium, glucose, calcium, phosphorus, parathyroid hormone, creatinine, creatinine clearance, estimated GFR (eGFR) by three equations (MDRD, CKDEPI and BIS1), urinalysis, and renal imaging (ultrasound). Additionally, HUGE equation was calculated from each volunteer applying the following equation (4):

$$
\begin{gathered}
\text { HUGE }=2.505458-[0.264418 \times \text { Hematocrit }]+[0.118100 \\
x \text { Serum Urea }(\mathrm{mg} / \mathrm{dl})][+1.383960 \text { if male }] \\
\text { HUGE }<0=\text { normal ageing kidney } \\
\text { HUGE } \geq 0=\text { chronic kidney disease }
\end{gathered}
$$

All the recruited patients were classified as having or not CKD after their evaluation by two independent nephrologists who based their diagnoses on the patient's medical record, blood and urine laboratories, as well as renal images, but were blind to the HUGE equation result and were no allowed to calculate it. This categorization in normal ageing kidney (NAK) or CKD was based on a coincident double blind nephrological evaluation which was considered to be the gold standard for diagnosing the presence or absence of chronic nephropathy (see clinical diagnosis criteria in Appendix).

Finally, the best strategies (most sensitive) were evaluated for screening CKD in young, old and very old population:

1) to use a single parameter (high HUGE or low eGFR); 2) to use a combination of two parameters (high HUGE and low eGFR); 3) to use a combination of three parameters (high HUGE, low eGFR and altered urinalyses).

It is worth mentioning that eGFR was obtained by the main GFR equations currently used (MDRD, CKD-EPI, or BIS1); and GFR cut-off value for young people was based on the lowest physiological GFR value $(80 \mathrm{~mL} /$ $\min / 1.73 \mathrm{~m}^{2}$ ), while the GFR cut-off value for elderly people was based on physiological GFR value based on Keller equation for old people $(60 \mathrm{~mL} / \mathrm{min} / 1.73$ $\left.\mathrm{m}^{2}\right)$, and for very old people $\left(45 \mathrm{~mL} / \mathrm{min} / 1.73 \mathrm{~m}^{2}\right)(7,8)$. The present study was approved by the Institutional Review Board and all participants provided their written informed consent.

\section{Results}

In this prospective study, HUGE equation accuracy was evaluated in 200 ambulatory clinical and nephrological patients (age range: 18-90) regularly assisted at Hospital Italiano de San Justo (Province of Buenos Aires, Argentina) since April 1st to September 30th (2016).

Table 1

\begin{tabular}{|c|c|c|c|c|}
\hline & $\begin{array}{l}\text { NAK }(\mathrm{n}: 56) \\
\text { Mean } \pm \text { SD }\end{array}$ & $\begin{array}{l}\text { CKD (n:48) } \\
\text { Mean } \pm \text { SD }\end{array}$ & $\mathbf{P}$ & $\begin{array}{c}\text { normal } \\
\text { values }\end{array}$ \\
\hline Age (years) & $76 \pm 7$ & $77 \pm 6$ & NS & -- \\
\hline Gender (male) & $15(33 \%)$ & $31(67 \%)$ & $<0.001$ & -- \\
\hline $\begin{array}{l}\operatorname{MDRD}(\mathrm{mL} / \\
\left.\min / 1.73 \mathrm{~m}^{2}\right)\end{array}$ & $74.9 \pm 16.8$ & $41.6 . \pm 22.2$ & $<0.001$ & $65 \pm 5^{*}$ \\
\hline $\begin{array}{l}\text { CKD-EPI (mL/ } \\
\left.\min / 1.73 \mathrm{~m}^{2}\right)\end{array}$ & $73.4 \pm 13.1$ & $40.7 \pm 21.8$ & $<0.001$ & $65 \pm 5^{*}$ \\
\hline $\begin{array}{l}\mathrm{BIS1}(\mathrm{mL} / \mathrm{min} / 1.73 \\
\left.\mathrm{m}^{2}\right)\end{array}$ & $63.4 \pm 12.2$ & $39.7 \pm 16.7$ & $<0.001$ & $65 \pm 5^{*}$ \\
\hline
\end{tabular}

Comparison between chronic kidney disease (CKD) and normal ageing kidney (NAK) elderly individuals: age, gender, MDRD, CKD-EPI, and BIS1 equations

Table 2

Comparison between chronic kidney disease (CKD) and normal ageing kidney (NAK) elderly individuals: hematocrit, uremia, creatininemia, urea, urinalysis, renal ultrasound, and HUGE equation

\begin{tabular}{lcccc}
\hline & $\begin{array}{c}\text { NAK (n:56) } \\
\text { Mean } \pm \text { SD }\end{array}$ & $\begin{array}{c}\text { CKD (n:48) } \\
\text { Mean } \pm \text { SD }\end{array}$ & p & $\begin{array}{c}\text { normal } \\
\text { values }\end{array}$ \\
\hline $\begin{array}{l}\text { Hematocrit }(\%) \\
\text { Serum urea (mg/ } \\
\text { dL) }\end{array}$ & $40.8 \pm 4.6$ & $38.3 \pm 4.5$ & 0.006 & $40-53$ \\
$\begin{array}{l}\text { Serum creatinine } \\
\text { (mg/dL) }\end{array}$ & $0.8 \pm 0.1$ & $1.9 \pm 0.9$ & $<0.001$ & $0.6-1$ \\
$\begin{array}{l}\text { Abnormal } \\
\text { Urinalysis (\%) }\end{array}$ & 0 & 100 & $<0.001$ & 0 \\
$\begin{array}{l}\text { Abnormal Renal } \\
\text { Ultrasound (\%) }\end{array}$ & 0 & 100 & $<0.001$ & 0 \\
HUGE & $-6.8 \pm 2.6$ & $-0.2 \pm 7.4$ & $<0.001$ & $<0$ \\
\hline
\end{tabular}

From this population ( $\mathrm{n}$ : 205), a coincident evaluation performed by two independent nephrologists (gold standard) determined that 85 individuals had normal ageing kidney (NAK) while 115 suffered from CKD. There was no diagnostic concordance between the nephrologists in 5 patients. Among the studied elderly people (n: 104), serum urea, serum creatinine, and HUGE values were significantly higher in CKD patients (n: 48), while eGFR value and hematocrit were significantly lower in CKD patients compared to NAK individuals (n: 56). Besides, renal 
ultrasounds and urinalyses were significantly abnormal in CKD patients compared to NAK (Tables 1 and 2). In this study, it was found that applying HUGE equation to the whole people, it had a sensitivity of $27.1 \%$ (IC: $17.9 \%-37.8 \%$ ), and a specificity of $100 \%$ (IC: $96.8-100 \%$ ).

\section{Table 3}

Correlation between chronic kidney disease (CKD) stages and HUGE value

\begin{tabular}{lcc}
\hline & $\begin{array}{c}\text { HUGE } \\
\text { median (range) }\end{array}$ & $\begin{array}{c}\text { Studied People } \\
\mathbf{n}(\%)\end{array}$ \\
\hline NAK & $-6.8(-8.5 /-5.1)$ & $115(58)$ \\
CKD Stage I & $-5.1(-7.5 /-3.7)$ & $11(5)$ \\
GFR: $\geq 90 \mathrm{~mL} / \mathrm{min}$ & $-3 .(-5.6 /-2.0)$ & $16(8)$ \\
CKD Stage II & & \\
GFR: 89-60 mL/min & $-3.5(-7.0 /-1.0)$ & $21(11)$ \\
CKD Stage III a & & $15(7)$ \\
GFR: 59-45 mL/min & $-2.9(-7.3 / 1.6)$ & $18(9)$ \\
CKD Stage III b & & \\
GFR: 44-30 mL/min & $0.7(-7.0 / 6.2)$ & $4(2)$ \\
CKD Stage IV & & \\
GFR: $29-15 \mathrm{~mL} / \mathrm{min}$ & $10.6(9.3-11.9)$ & \\
CKD Stage V & & \\
GFR $<15 \mathrm{~mL} / \mathrm{min}$ & & \\
\hline
\end{tabular}

NAK: normal ageing kidney, GFR: glomerular filtration rate

Table 4

Performance of different strategies for CKD screening in young population

\begin{tabular}{lcc}
\hline & $\begin{array}{c}\text { SENSITIVITY } \\
(\%)(95 \% \text { CI) }\end{array}$ & $\begin{array}{c}\text { ESPECIFICITY } \\
(\%)(95 \% \text { CI) }\end{array}$ \\
\hline high HUGE & $8.6(1.8-23.1)$ & $100(93.3-100)$ \\
low GFR (MDRD) & $60.0(42.1-76.1)$ & $66.7(52.1-79.2)$ \\
low GFR (CKD-EPI) & $61.8(43.5-77.8)$ & $86.8(74.7-94.5)$ \\
low GFR (BIS1) & $56.7(37.4-74.5)$ & $81.4(66.6-91.6)$ \\
high HUGE or low GFR (MDRD) & $60.0(42.0-76.0)$ & $86.8(74.6-94.4)$ \\
high HUGE or low GFR (CKD-EPI) & $60.0(42.1-76.1)$ & $86.8(74.7-94.5)$ \\
high HUGE or low GFR (BIS1) & $48.0(31.4-66.0)$ & $84.9(72.4-93.3)$ \\
$\begin{array}{l}\text { high HUGE or altered urinalysis } \\
\text { high HUGE or low GFR (MDRD) or }\end{array}$ & $51.3(33.9-68.6)$ & $100(93.3-100)$ \\
$\begin{array}{l}\text { altered urinalysis } \\
\text { high HUGE or low GFR (CKD-EPI) or } \\
\text { altered urinalysis }\end{array}$ & $86.8(69.7-95.2)$ & $67.9(53.7-80.1)$ \\
\hline
\end{tabular}

high HUGE or low GFR (BIS1) or $80.0(63.1-91.6) \quad 84.9(72.4-93.3)$ altered urinalysis

GFR: estimated glomerular filtration rate. 95\% CI: confidence interval. high HUGE: HUGE $\geq 0$, low GFR: GFR $<80 \mathrm{ml} / \mathrm{min} / 1.73 \mathrm{~m}^{2}$
Table 5

Performance of different strategies for CKD screening in the elderly

\begin{tabular}{lcc}
\hline & $\begin{array}{c}\text { SENSITIVITY (\%) } \\
\mathbf{( 9 5 \% ~ C I ) ~}\end{array}$ & $\begin{array}{c}\text { ESPECIFICITY (\%) } \\
\text { (95\% CI) }\end{array}$ \\
\hline high HUGE & $40.0(26.4-54.8)$ & $100.0(94.2-100)$ \\
low GFR (MDRD) & $83.3(69.8-92.5)$ & $77.8(64.4-87.9)$ \\
low GFR (CKD-EPI) & $83.3(69.8-92.5)$ & $78.6(65.6-88.4)$ \\
low GFR (BIS1) & $85.4(72.2-93.9)$ & $55.6(41.4-69.1)$ \\
high HUGE or low GFR (MDRD) & $83.3(69.8-92.5)$ & $77.9(64.4-87.9)$ \\
$\begin{array}{l}\text { high HUGE or low GFR (CKD- } \\
\text { EPI) }\end{array}$ & $83.3(69.8-92.5)$ & $78.6(65.6-88.4)$ \\
high HUGE or low GFR (BIS1) & $85.4(72.2-93.9)$ & $57.1(43.2-70.3)$ \\
$\begin{array}{l}\text { high HUGE or altered urinalysis } \\
\text { high HUGE or low GFR MDRD } \\
\text { or altered urinalysis }\end{array}$ & $56.2(41.2-70.5)$ & $100.0(93.6-100)$ \\
$\begin{array}{l}\text { high HUGE or low CKD-EPI or } \\
\text { altered urinalysis }\end{array}$ & $95.8(85.7-99.5)$ & $78.6(65.5-88.4)$ \\
$\begin{array}{l}\text { high HUGE or low GFR BIS1 or } \\
\text { altered urinalysis }\end{array}$ & $97.9(88.9-99.9)$ & $78.5(65.6-88.4)$ \\
\hline $\begin{array}{l}\text { GFR: estimated glomerular filtration rate, IC: interval of confidence, high } \\
\text { HUGE: HUGE } \geq 0, \text { low GFR: GFR }<60 \mathrm{~mL} / \mathrm{min} / 1.73 \mathrm{~m}^{2}\end{array}$
\end{tabular}

Table 6

Performance of triple combined strategy (HUGE-eGFRurinalyses) in old and very old individuals

\begin{tabular}{|c|c|c|}
\hline & $\begin{array}{l}\text { SENSITIVITY (\%) } \\
(95 \% \mathrm{CI})\end{array}$ & $\begin{array}{c}\text { ESPECIFICITY }(\%) \\
(95 \% \mathrm{CI})\end{array}$ \\
\hline $\begin{array}{l}\text { Old People high HUGE or low } \\
\text { BIS1 or altered urinalysis }\end{array}$ & $100(89.7-100)$ & $76.3(59.8-88.6)$ \\
\hline $\begin{array}{l}\text { Very Old People high HUGE or } \\
\text { low GFR BIS1 or altered urinalysis }\end{array}$ & $92.9(66.1-99.8)$ & $61.1(35.7-82.7)$ \\
\hline
\end{tabular}

However, since it had already been reported that HUGE equation showed better performance for screening CKD in elderly people, its accuracy was also evaluated in the aged group (n: 112); and It was found that HUGE equation had a sensitivity of $40.0 \%$ (IC: $26.4 \%-54.8 \%$ ), and a specificity of $100 \%$ (IC: $94.2 \%$ - $100 \%$ ) for screening CKD in the elderly. Moreover, there was no significant difference in HUGE performance between old (age $\geq 65$ and $<80$ years old) and very old (age $\geq 80$ years old) individuals. Correlation between GFR level (based on MDRD) and HUGE value was evaluated; and a significant and inverse correlation between them in this studied group of patients was documented: Sperman correlation: 0.5, p: $<0.001$ (Table 3). Additionally, since NAK and advanced CKD share the characteristic of presenting a reduced GFR, the HUGE equation's accuracy was also evaluated to distinguish between NAK and stages IIIb-V CKD (GFR < 45 mL/ $\mathrm{min} / 1.73 \mathrm{~m}^{2}$ ) in the elderly (n: 104). It was documented that HUGE equation had a sensitivity of $56.7 \%$ (IC: 
$37.4 \%-74.5 \%$ ), and a specificity of $95.9 \%$ (IC: $88.6 \%$ - 99.1\%) for screening CKD in elderly individuals who have an estimated GFR $<45 \mathrm{~mL} / \mathrm{min} / 1.73 \mathrm{~m}^{2}$. Finally, it was evaluated what it would happen if any of the studied subjects has at least one of the following parameters (HUGE, eGFR, urinalyses) regarding their accuracy for screening CKD. It was documented that a combined strategy (HUGE-eGFR-urinalysis) had a better performance than using any of these parameters separately, independently of the applied GFR equation (MDRD, CKD-EPI or BIS1). Moreover, it was found that the combination of HUGE - eGFR (MDRD/CKDEPI)-urinalysis was the best strategy for screening CKD in young subjects (Table 4), while the combination of HUGE-eGFR(BIS1)-urinalysis was the best strategy in old subjects (Tables 5). Even more, it was documented that these CKD screening combined strategies showed a better performance in the elderly when the GFR cut-off applied was in accordance to the patient's age: $60 \mathrm{~mL} / \mathrm{min} / 1.73$ $\mathrm{m}^{2}$ for old subjects, and $45 \mathrm{~mL} / \mathrm{min} / 1.73 \mathrm{~m}^{2}$ for very old subjects (Table 6).

\section{Discussion}

As far as we know, this is the first study which prospectively evaluated HUGE equation performance out of Spain. As it was documented in the Spanish study which originally described HUGE equation, and also in our previous retrospective study regarding this equation, we found that HUGE equation showed a better performance in elderly people (eGFR $<60 \mathrm{~mL} / \mathrm{min} / 1.73$ $\mathrm{m}^{2}$ ). Even though, a significantly lower sensitivity (40.0\%) was documented in HUGE performance in this prospective study, compared to the original HUGE study $(92.8 \%)$, although it should be taken into account that equations usually show better performance in the population where they have originally been developed (4, 5 , 9). However, it was documented that HUGE equation showed an adequate performance in elderly people with a GFR $\leq 45 \mathrm{~mL} / \mathrm{min} / 1.73 \mathrm{~m}^{2}$ : sensitivity $56.7 \%$ (96\% CI: $37.4 \%-74.5 \%)$, and a specificity of $95.9 \%(95 \%$ CI: $88.6 \%-99.1 \%$ ). Additionally, it was found that the screening strategy of combining HUGE with other simple variables, such as eGFR and urinalyses, showed a very good performance for detecting CKD, not only in elderly people but also in young people. The best combined CKD screening strategy was to consider a potential chronic nephropathy patient any young individual who showed a high HUGE, or a low eGFR (based on MDRD or CKDEPI), or an altered urinalyses; and any old subject who showed a high HUGE, or a low eGFR (based on BIS1), or an altered urinalyses. Both triple combined CKD screening strategies showed a sensitivity around $86 \%$ and $98 \%$, in young and elderly people, respectively (Tables 4 , 5 and 6). This finding makes sense since HUGE equation is blind to two important and simple to obtain variables for diagnosing CKD: GFR and urinalyses. Thus, to join these two variables to the CKD screening (triple strategy) improves the opportunity of detecting individuals suffering from chronic nephropathy. Besides, MDRD and CKD-EPI are currently the best GFR equations for the young, while BIS1 equation is the best GFR equation (validated) for the elderly (8). Regarding HUGE equation performance in the elderly group, its specificity was 100\% (no false positive cases), while it misclassified 26 CKD individuals as not having renal disease. Among these false negative cases, most of them (n: 13) were induced by a slightly high serum urea level, six to a slightly high serum urea and slightly low hematocrit values, five induced by abnormal urinalyses, and two induced by altered renal ultrasound.

Regarding performance of the triple combined CKD screening strategy (HUGE-eGFR-urinalyses) in old population (65 - 79 years old), it had no false negative case, while it had nine false positive cases. Among these false positive cases, most of them (n: 8 ) were induced by a low normal (borderline) GFR value, and the other (n: 1) was induced by a marked non renal anemia. Finally, regarding the performance of the triple combined CKD screening strategy (HUGE-eGFR-urinalyses) in very old population (age $\geq 80$ years old), it had one false negative case due to an abnormal renal ultrasound, while it had seven false positive cases. Among these false positive cases, most of them (n: 4) were secondary to low normal (borderline) GFR value, and the rest (n: 3) were induced by a marked non renal anemia.

\section{Conclusion}

This prospective study documented that the HUGE equation had and acceptable performance for screening moderate-severe CKD (stages IIIb-V) in elderly people, and an excellent performance for screening CKD in general population in combination with estimated GFR and urinalyses.

Conflict of Interest: All the authors declare that they have no conflict of interest.

Ethical approval: All procedures performed in studies involving human participants were in accordance with the ethical standards of the institutional and or national research committee and with the 1964 Helsinki declaration and its later amendments or comparable ethical standards.

Informed consent: Informed consent was obtained from all the participants included in the study.

\section{Appendix}

CKD diagnosis: to have at least an abnormal value in one of the following parameters: GFR, urinalysis, and renal ultrasound

- Reduced GFR: a GFR value lower than the expected one secondary to ageing: expected GFR $=130$ - age $(\mathrm{mL} / \mathrm{min} / 1.73$ $\left.\mathrm{m}^{2}\right)$

- Abnormal urinalysis: presence of renal (dysmorphic) hematuria ( $>3$ red blood cells) and / or proteinuria ( $>0.2 \mathrm{~g} /$ day)

- Abnormal renal ultrasound: presence of at least one of the following alterations in renal parenchyma: reduced size, asymmetry, increased parenchyma echogenicity, many cysts, altered cortex-medulla proportion. 


\section{References}

1. Clarkson M, Magee C, Brenner B. The kidney. Philadelphia. Saunders. 2010.

2. Musso CG, Macías Nuñez JF, Oreopoulos DG. Physiological similarities and differences between renal aging and chronic renal disease. J Nephrol. 2007:20(5):586-7

3. Musso CG, Jauregui JR. How to differentiate renal senescence from chronic kidney disease in clinical practice.Postgrad Med. 2016;128(7):716-21

4. Álvarez-Grégori J, Robles N, Mena C, Ardanuy R, Jauregui J, Macías Nçuñez JF. The value of a formula including haematocrit, blood urea and gender (HUGE) as a screening test for chronic renal insufficiency. The Journal of Nutrition, Health Aging.2011;15(6)2011:1-5
5. Musso CG, de Los Rios E, Vilas M, Terrasa S, Bratti G, Varela F, Diez GR, Jauregui J, Luna D.The HUGE formula (hematocrit, urea, gender) for screening for chronic kidney disease in elderly patients: a study of diagnostic accuracy.Int Urol Nephrol. 2016 Dec 29. doi: 10.1007/s11255-016-1486-6

6. Naughton C. Drug-Induced Nephrotoxicity. American Family Physician. 2008; 78 (6):743-750

7. Rennke H, Denker B. Renal Pathophysiology. Philadelphia. Lippincott Williams \& Wilkins. 1994: 267-290

8. Musso CG, Álvarez-Gregori J, Jauregui J, Macías-Núñez JF.Glomerular filtration rate equations: a comprehensive review.Int Urol Nephrol. 2016;48(7):1105-10

9. Dawson-Saunders B, Trapp R. [Medical Biostatistics. Mexico. Manual Moderno].1993:51-52 